\title{
Nonlinear effects in Thomson backscattering
}

\author{
C. Maroli, V. Petrillo, ${ }^{*}$ P. Tomassini, and L. Serafini \\ Università degli Studi, INFN Milano, Via Celoria, 1620133 Milano, Italy
}

(Received 19 October 2012; published 27 March 2013)

\begin{abstract}
We analyze the nonlinear classical effects of the $\mathrm{X} / \gamma$ radiation produced by Thomson/Compton sources. We confirm the development of spectral fringes of the radiation on axis, which comports broadening, shift, and deformation of the spectrum. For the nominal parameters of the SPARC-LAB Thomson scattering and of the European Proposal for the gamma source ELI-NP, however, the radiation, when collected in the suitable acceptance angle, does not reveal many differences from that predicted by the linear model and the nonlinear redshift is subdominant with respect to the quantum recoil. An experiment aimed to the study of the nonlinearities is proposed on the SPARC-LAB source.
\end{abstract}

DOI: 10.1103/PhysRevSTAB.16.030706

PACS numbers: 41.60.Ap

\section{INTRODUCTION}

The availability of high-intensity laser pulses and highbrightness electron beams generated by linear accelerators has given impulse to the project and development of Thomson/Compton [1-4] sources, which rely on the backscattering between the laser light and the relativistic particle bunch. The radiation obtained in this way is in the range of $\mathrm{x}$ and $\gamma$ rays and has properties of wide tunability, high spectral density, ultrashort time duration, and large degree of transverse coherence. These characteristics rend the Thomson/Compton sources suitable for several applications such as advanced imaging techniques [5,6] and nuclear resonant fluorescence, resulting in a valid alternative to synchrotrons [7], free-electron lasers [8-11], and highorder harmonic generation in gases [12].

The Thomson/Compton X-source PlasmonX SPARCLAB [13-15] at the National Laboratory of Frascati (INFN-LNF) is based on the backscattering of the light pulse of the intense Ti:sapphire laser FLAME [16] with the high-brightness electron beam produced by the photoinjector SPARC [17]. The final electron beam energy $E_{e}$ at the exit of the linac ranges between 30 and $270 \mathrm{MeV}$, thereby permitting the production of Doppler blueshifted hard $\mathrm{x}$ rays with wavelength $\lambda_{T} \approx \lambda_{L} /\left(4 \gamma^{2}\right) \quad\left(\lambda_{L}=\right.$ $800 \mathrm{~nm}$ is the wavelength of FLAME and the Lorentz factor of the electrons $\gamma \approx 60$ ) well below $1 \AA$, corresponding to photon energies $E_{\mathrm{ph}}=h c / \lambda_{T}$ ( $h$ is the Planck constant) between 20 and $500 \mathrm{KeV}$.

Since the radiation of Thomson/Compton sources covers a large frequency range, within the framework of the Extreme Light Infrastructure (ELI) project, a Compton $\gamma$ rays source is foreseen. The first possible electron working

\footnotetext{
*petrillo@mi.infn.it
}

Published by the American Physical Society under the terms of the Creative Commons Attribution 3.0 License. Further distribution of this work must maintain attribution to the author(s) and the published article's title, journal citation, and DOI. points studied by the European Proposal for ELI-NP [18] will be between 360 and $750 \mathrm{MeV}$. Colliding with laser photons of $2.4 \mathrm{eV}$, these beams will generate $\gamma$ photons of wavelengths shorter than $10^{-13} \mathrm{~m}$ with energies up to $20 \mathrm{MeV}$.

In all $\mathrm{X} / \gamma$ source implementations, a particularly important issue is the dimension of the spectral bandwidth. While in the biomedical imaging field relative bandwidths of few per cent are considered viable, in the nuclear applications of $\gamma$ rays the requirements are much more demanding and this value drops down to the order of $10^{-3}$, so that the maximum care must be payed in taking under control the band enhancement. The most important factors that contribute to enlarge the spectrum are acceptance, electron emittance, and energy spread as well as diffraction, natural bandwidth, and time duration of the laser pulse, while, instead, collective effects tend to decrease it [19]. Nonlinear effects are another significant cause of bandwidth broadening. When the laser intensity is increased, various nonlinear phenomena emerge in the description of the electron trajectories and the consequent radiation. Nonlinear effects will therefore play key roles in future tabletop devices and $\mathrm{x} / \boldsymbol{\gamma}$-ray sources, where several Joules of laser energy will be focused on spot sizes of few wavelengths, with normalized laser parameters $a_{0}=\frac{\lambda_{L} e E_{0}}{2 \pi m c^{2}}$ close or beyond one ( $E_{0}$ is the peak value of the electric field, $e$ and $m$ the electron charge and rest mass, and $c$ the speed of light).

At electron energies below $1 \mathrm{GeV}$ several characteristics of the radiation can be evaluated by means of the wellknown classical procedure [20-22].

Classical nonlinear effects concur with the quantum recoil in changing the resonant wavelength. They induce shifts and deformations of the spectrum on the fundamental frequency and the growth of harmonics. They can be calculated in closed form under the hypothesis that the laser is a plane wave [13,23]. The presence of a well defined longitudinal amplitude modulation [24,25] has been introduced to evaluate the distortions on the 
fundamental and the rise of harmonics. Other approaches consist in evaluating the shift by means of the decrease in velocity of the electron due to the ponderomotive action of the laser field [26], or in calculating numerically the trajectories and inserting them into the classical radiation integral $[25,27,28]$.

In this paper, we study the occurrence of the nonlinear effects in the classical Thomson electron-photon interaction. We have investigated realistic situations, covering the case of PlasmonX, where the Ti:sapphire laser (5 J, $6 \mathrm{ps}$ ) interacts with electron beams of relatively low energy and the case of the European proposal for ELI-NP [29], where the electrons are significantly more energetic. The dependence of the nonlinear terms on the intensity of the laser pulse and the consequent spectral distortions on axis, appearing in the form of a sequence of fringes, have been analyzed. We have applied the stationary phase method to the integral expression of the double differential spectrum and given a closed form of it in terms of standard transcendental functions. With the aid of this analytical formula, we have connected the occurrence of the fringes to a chirp in the emission due to the variation of the electron Lorentz factor along the trajectory. We have provided the $\mathrm{X} / \gamma$ spectral distributions, weighted the importance of the nonlinear effects on the bandwidth, and compared the nonlinear shift in wavelengths to the quantum shift due to the electron recoil. The shape of the radiation spectrum produced on axis by a single electron appears affected by nonlinearities, as already presented in Ref. [3]. We show, on the contrary, that the integration over the acceptance angle and the extension to realistic electron beams permit one to exclude a substantial enhancement of the bandwidth in the range of parameters of both PlasmonX and ELI-NP sources, ensuring that a very thin bandwidth can be achieved. This conclusion is very important in view of the future applications of this kind of source. Since the problem of the nonlinear enhancement of the bandwidth is important in Thomson/Compton sources, and since the commissioning of PlasmonX is imminent, at the end of the paper, we propose and quantify a determinant experiment on the SPARC-LAB source for studying the nonlinear characters of the radiation spectrum.

\section{THE MODEL EQUATIONS}

The double differential energy spectrum vs the frequency $\omega$ and solid angle $\Omega$, as well as the photon number at all points of the collector, are given by the well-known radiation integral [20]:

$$
\begin{aligned}
\frac{d^{2} W}{d \omega d \Omega} & =\hbar \omega \frac{d^{2} N}{d \omega d \Omega} \\
& =\frac{e^{2}}{4 \pi^{2} c}\left|\int_{-\infty}^{+\infty} d t e^{i \omega t} \frac{\underline{n} \times\left\{\left[\underline{n}-\underline{\beta}\left(t^{\prime}\right)\right] \times \underline{\dot{\beta}}\left(t^{\prime}\right)\right\}}{\left[1-\underline{n} \cdot \underline{\beta}\left(t^{\prime}\right)\right]^{3}}\right|^{2},
\end{aligned}
$$

where $\underline{n}$ is the unit vector of the emitted radiation (i.e., the direction of the observer), $\beta$ is the normalized electron velocity, $\underline{\dot{\beta}}$ its acceleration, $t^{\prime}=t-\frac{1}{c}\left|\underline{n} r-\underline{r}\left(t^{\prime}\right)\right|$ the retarded time and cgs units are used throughout. Equation (1) permits the calculation of the total number of photons collected on the screen vs frequency

$$
\frac{d N}{d \omega}=\int d \Omega \frac{d^{2} N}{d \omega d \Omega}
$$

as well as the (overall) photon number in one interaction:

$$
N=\int d \omega d \Omega \frac{d^{2} N}{d \omega d \Omega} .
$$

The laser pulse is described by the usual complete paraxial form [30]. The classical radiation reaction is considered negligible and is not taken into account in the equations of motion.

We consider the case of one electron subject to the laser action in the backscattering situation and in a head-on collision with the laser pulse. The equations of motion have the form

$$
\begin{aligned}
& \frac{d p_{x}}{d t^{\prime}}=-K g\left(t^{\prime}\right)\left(1+\frac{p_{z}}{\gamma}\right), \\
& \frac{d p_{y}}{d t^{\prime}}=0, \quad \frac{d p_{z}}{d t^{\prime}}=K g\left(t^{\prime}\right) \frac{p_{x}}{\gamma},
\end{aligned}
$$

where $\underline{p}=\gamma \underline{\beta}$ and $K=e E_{0} / m c$. The electron starts at $t^{\prime}=0$ from a point of coordinates $x(0)=0, y(0)=0$ and $z(0)$ a (large) negative number. The initial value of the electron momentum is $\underline{p}=\left(0,0, p_{0}\right)$ with $p_{0}=\gamma_{0} \beta_{z 0}>0$, where $\beta_{z 0}=\beta_{z}(0)$ and $\gamma_{0}=\gamma(0)$. The function $g\left(t^{\prime}\right)$ appearing in Eq. (4) is directly connected to the form of the laser pulse which is supposed to be polarized along the $x$ axis and to move with velocity $c$ on the (negative) $z$ axis. The laser electric field is written as

$$
\begin{aligned}
\underline{E}(x, y, z, t) & =E_{0} g(x, y, z, t) \underline{e}_{x} \\
& =E_{0} G(x, y, z, t)\left(\sin \Gamma-\frac{z}{z_{R}} \cos \Gamma\right) \underline{e}_{x},
\end{aligned}
$$

where

$$
\begin{gathered}
G(x, y, z, t)=\frac{A\left(z+c t+\frac{z(0)}{\beta_{z 0}}\right)}{1+\frac{z^{2}}{z_{R}^{2}}} e^{-\sigma_{\perp}^{2}\left\{\left(x^{2}+y^{2}\right) /\left[1+\left(z^{2} / z_{R}^{2}\right)\right]\right\}, \quad(6)} \\
\Gamma(x, y, z, t)=k_{L}\left(z+c t+\frac{z(0)}{\beta_{z 0}}\right)+\sigma_{\perp}^{2}\left(x^{2}+y^{2}\right) \frac{z z_{R}}{z^{2}+z_{R}^{2}}
\end{gathered}
$$

and $k_{L}$ and $\omega_{L}=c k_{L}$ are the laser wave number and angular frequency. The function $g\left(t^{\prime}\right)$ appearing in (4) is $g\left(t^{\prime}\right) \equiv g\left[x\left(t^{\prime}\right), y\left(t^{\prime}\right), z\left(t^{\prime}\right)\right]$, where $x\left(t^{\prime}\right), y\left(t^{\prime}\right), z\left(t^{\prime}\right)$ give the instantaneous position of the electron, $z_{R}=k / 2 \sigma_{\perp}^{2}$ is the Rayleigh length, and $\sigma_{\perp}=\frac{2}{w_{0}}, w_{0}$ being the $\mathrm{rms}$ spot 
diameter. The function $A>0$ is an arbitrary, slowly varying function which depends on the form of the longitudinal laser pulse modulation. In the Gaussian case $A(z+c t+$ $\left.\frac{z(0)}{\beta_{z 0}}\right)=e^{-\sigma_{\|}^{2}\left\{z+c t+\left[z(0) / \beta_{z 0}\right]\right\}^{2}}$, where $\sigma_{\|}=\frac{\sqrt{2}}{c \Delta \tau}$ and $\Delta \tau$ is the pulse rms time duration. We are assuming, as can be seen from (6), that the laser waist is at $z=0$, while the peak value of the pulse at the initial time is at $z=-\frac{z(0)}{\beta_{z 0}}$, so that the interaction with the electron is taking place around $z=0$ at the somewhat later time $t_{0}=-\frac{z(0)}{c \beta_{z 0}}>0$ with the laser at its maximum focusing. As in Ref. [24], we shall consider the spectrum only on the $z$ axis, i.e., the unit vector $\underline{n}$ in (1) is $\underline{n}=\underline{e}_{z}$.

\section{A. Explicit form of the spectrum}

The equations of motion (4) are more conveniently integrated by using the phase

$$
\varphi\left(t^{\prime}\right)=k_{L}\left[z\left(t^{\prime}\right)+c\left(t^{\prime}-t_{0}\right)\right]
$$

as the new independent variable, with $\varphi(0)=\varphi_{0}=$ $k_{L}\left[z(0)-c t_{0}\right]$. Likewise, if we change from the laboratory time $t$ to the retarded time $t^{\prime}$ and then again to the variable $\varphi$ in the integral (1), we can write the double differential spectrum in the closed form:

$$
\frac{d^{2} W}{d \omega d \Omega}=\frac{e^{2} a_{0}^{2}}{16 \pi^{2} c \chi_{0}^{2}}\left|I_{+}+I_{-}\right|^{2},
$$

where $a_{0}=e E_{0} / m c \omega_{L}$ is the laser parameter, $\chi_{0}=$ $\gamma_{0}\left(1+\beta_{z 0}\right)$ and

$$
I_{ \pm}=\int_{-\infty}^{\infty} d \varphi u(\varphi)\left(\frac{z}{z_{R}} \pm i\right) G(\varphi) e^{\left(i / \omega_{l i n}\right) \Phi_{ \pm}(\varphi)},
$$

where

$$
\begin{gathered}
u(\varphi)=\frac{2-2 \chi_{0} \gamma(\varphi)+\chi_{0}^{2}}{\left[2 \gamma(\varphi)-\chi_{0}\right]^{2}}, \\
\gamma(\varphi)=\gamma_{0}+\frac{a_{0}^{2} F^{2}(\varphi)}{2 \chi_{0}}, \\
\Phi_{ \pm}=\left(\omega \pm \omega_{l i n}\right) \varphi+a_{0}^{2} \omega H(\varphi) \\
\pm \sigma_{\perp}^{2} \omega_{l i n} \frac{x^{2}(\varphi) z(\varphi) / z_{R}}{1+z^{2}(\varphi) / z_{R}^{2}}, \\
H(\varphi)=\int_{\varphi_{0}}^{\varphi} d \varphi^{\prime} F^{2}\left(\varphi^{\prime}\right), \\
F(\varphi)=\int_{\varphi_{0}}^{\varphi} d \varphi^{\prime} g\left(\varphi^{\prime}\right),
\end{gathered}
$$

the last two transcendental functions depending on the form of the laser pulse modulation along the direction of propagation and $x(\varphi)$ and $z(\varphi)$ are known from the electron trajectory. The frequency

$$
\omega_{\text {lin }}=\omega_{L} \frac{1+\beta_{z 0}}{1-\beta_{z 0}}=\omega_{L} \chi_{0}^{2}
$$

is the usual Doppler shifted resonant frequency on axis.

\section{B. Gaussian laser pulse with $w_{0} \gg \lambda_{L}$}

In the case of a Gaussian laser pulse with a very large spot diameter $w_{0} \gg \lambda_{L}$ (and therefore very long Rayleigh length $z_{R}$ ), the integrals in (9) assume the form

$$
I_{ \pm}= \pm i \int_{-\infty}^{\infty} d \varphi u(\varphi) e^{-\epsilon^{2} \varphi^{2}+\left(i / \omega_{\operatorname{lin}}\right)\left[\left(\omega \pm \omega_{\text {lin }}\right) \varphi+a_{0}^{2} \omega H(\varphi)\right]},
$$

while the transcendental function $F$ becomes

$$
F(\varphi)=\int_{\varphi_{0}}^{\varphi} d \varphi^{\prime} \sin \varphi^{\prime} e^{-\epsilon^{2} \varphi^{\prime 2}}
$$

with $\epsilon=\sqrt{2} / \omega_{L} \Delta \tau$. If $\epsilon \ll 1$ and $\varphi_{0} \rightarrow-\infty$ the function $F$ assumes the approximate expression:

$$
F(\varphi) \approx-\cos \varphi e^{-\epsilon^{2} \varphi^{2}}
$$

and therefore

$$
H(\varphi) \approx \frac{1}{4 \epsilon} \sqrt{\frac{\pi}{2}}[1+\operatorname{erf}(\sqrt{2} \epsilon \varphi)]+\frac{1}{4} \sin 2 \varphi e^{-2 \epsilon^{2} \varphi^{\prime 2}} .
$$

The function $u(\varphi)$ can be approximated with its linear value $u_{0}$ :

$$
u_{0}=\frac{1+\beta_{z 0}}{1-\beta_{z 0}}=\chi_{0}^{2}
$$

and, by changing to the slow variable $y=\epsilon \varphi$ and using the Bessel identity

$$
e^{i x \sin \theta}=\sum_{n=-\infty, \ldots \infty} J_{n}(x) e^{i n \theta}
$$

we get

$$
I_{ \pm} \cong \frac{u_{0}}{\epsilon} \sum_{n=-\infty, \ldots \infty} \mathfrak{\Im}_{ \pm, n}
$$

where

$$
\Im_{ \pm, n}= \pm i \int_{-\infty}^{\infty} d y e^{-y^{2}} J_{n}\left(\frac{a_{0}^{2} \omega}{4 \omega_{\operatorname{lin}}} e^{-2 y^{2}}\right) e^{\left(i \varrho_{ \pm, n} / \epsilon \omega_{\text {lin }}\right)}
$$

with

$$
\varrho_{ \pm, n}(y)=\left[\left[\omega+(2 n \pm 1) \omega_{\operatorname{lin}}\right] y+\frac{a_{0}^{2} \omega}{4} \sqrt{\frac{\pi}{2}} \operatorname{erf}(\sqrt{2} y)\right] .
$$

When $\epsilon \ll 1$, the integrals in the previous equation (24) can be approximated by using the method of stationary phase, applied to those phases $\varrho_{ \pm, n}$ that possess stationary values over the range of integration. We can write at last 


$$
\frac{d^{2} W}{d \omega d \Omega}=\frac{e^{2} a_{0}^{2} \chi_{0}^{2}}{16 \pi^{2} c \epsilon^{2}}\left|\sum_{n=1, \ldots, \infty} \Re_{n}(\omega)\right|^{2}
$$

where

$$
\Re_{n}(\omega)=(-)^{n} \int_{-\infty}^{\infty} d y \Delta_{n}(y) e^{-y^{2}+\left[i\left(\varrho_{+, n}-4 n \omega_{l i n} y\right) / \epsilon \omega_{l i n}\right]},
$$

and

$$
\Delta_{n}(y)=J_{n}\left(\frac{a_{0} \omega}{4 \omega_{\text {lin }}} e^{-2 y^{2}}\right)+J_{n-1}\left(\frac{a_{0} \omega}{4 \omega_{\text {lin }}} e^{-2 y^{2}}\right) .
$$

The integrals $\Re_{n}(\omega)$ assume large values only within the frequency bands:

$$
\omega_{\operatorname{lin}} \frac{(2 n-1)}{1+a_{0}^{2} / 2}<\omega<(2 n-1) \omega_{\operatorname{lin}}(n=1,2, \ldots) .
$$

\section{The fundamental frequency interval $n=1$}

If the frequency bands around the single odd harmonic frequencies $\omega_{n}=(2 n-1) \omega_{\text {lin }}$ do not superpose, or the superposition is unimportant, the double differential spectrum within the fundamental frequency region can simply be written as

$$
\frac{d^{2} W}{d \omega d \Omega}=\frac{e^{2} a_{0}^{2} \chi_{0}^{2}}{16 \pi^{2} c \epsilon^{2}}\left|\Re_{1}(\omega)\right|^{2},
$$

where

$$
\Re_{1}=\int_{-\infty}^{\infty} d y\left[J_{0}+J_{1}\right] e^{-y^{2}+i\left(\varrho_{+, 1}-4 \omega_{\text {lin }} y\right) / \epsilon \omega_{\text {lin }}}
$$

and the argument of the Bessel functions is $\left(\omega-\omega_{\operatorname{lin}}\right) /$ $\left(2 \omega_{\text {lin }}\right)$. In the linear limit $\left(a_{0} \ll 1\right)$, the double differential spectrum becomes

$$
\frac{d^{2} W}{d \omega d \Omega}=\frac{e^{2} a_{0}^{2} \gamma_{0}^{2}\left(1+\beta_{z 0}\right)^{2}}{16 \pi c \epsilon^{2}} e^{-\Delta \tau^{2}\left(\omega-\omega_{\text {lin }}\right)^{2} / 4 \gamma_{0}^{4}\left(1+\beta_{z 0}\right)^{4}} .
$$

If $a_{0}$ is a very small number, the spectrum has a purely Gaussian form around the linear resonance frequency $\omega_{\text {lin }}$ as given in (16). The other two basic quantities determining the form of the spectrum are $\gamma_{0}$ and the paraxial large number $\omega_{L} \Delta \tau$. When $\gamma_{0}$ increases, $\omega_{\text {lin }}$ increases as $\gamma_{0}^{2}$ and the spectrum moves toward higher frequencies, while the width and the peak value of the Gaussian increase. Likewise, when the time duration $\Delta \tau$ of the laser pulse increases, the Gaussian spectrum becomes more and more narrow with its peak value increasing. It is worth noting that, according to (31), the two parameters $a_{0}$ and $\omega_{L} \Delta \tau$ act with contrary trend. We therefore should expect nonlinear effects not only in those cases when $a_{0}=\frac{e E_{0}}{\omega_{L} m c}=$ $4.3 \frac{\lambda_{L}}{w_{0}} \sqrt{\left(1+\beta_{z 0}\right)^{2} \frac{E(J)}{\Delta \tau(p s)}}$ is of the order of or larger than 1 , but also when $a_{0}<1$ and the paraxial number $\omega_{L} \Delta \tau$ is sufficiently large, to compensate at least partially for the smallness of $a_{0}$ [26]. The stationary values of the phase in Eq. (30) $y_{\mathrm{st}}(\omega)$ are given by

$$
e^{-2 y_{\mathrm{st}}^{2}}=\frac{\omega_{\operatorname{lin}}-\omega}{\omega a_{0}^{2} / 2}
$$

where $\omega$ must satisfy relation (28) with $n=1$.

For each value of the frequency inside this range, the phase presents two stationary values. They tend to superpose when $\omega$ approaches the limit value $\omega_{\text {lin }} /\left(1+a_{0}^{2} / 2\right)$ from the right, thereby giving rise to a stationary value of higher order [31,32].

Considering only the frequency interval where the two stationary points $\pm y_{\mathrm{st}}(\omega)$ are well separated, we can write the following expression:

$$
\left[\frac{d^{2} W}{d \omega d \Omega}\right]_{\text {approx }}=\frac{e^{2} \chi_{0}^{2}}{4 \pi c \epsilon} M(\omega) \cos ^{2}[\psi(\omega)]
$$

with

$$
\begin{gathered}
M(\omega)=\frac{\omega_{\text {lin }}}{\omega} \frac{\left[J_{0}+J_{1}\right]^{2}}{y_{\mathrm{st}}} \\
\psi(\omega)=\frac{1}{\epsilon}\left[\left(\frac{\omega}{\omega_{\text {lin }}}-1\right) y_{\mathrm{st}}+\frac{a_{0}^{2} \omega}{4 \omega_{\mathrm{lin}}} \sqrt{\frac{\pi}{2}} \operatorname{erf}\left(\sqrt{2} y_{\mathrm{st}}\right)\right]-\frac{\pi}{4} \\
y_{\mathrm{st}}(\omega)=\sqrt{-\ln \left(\frac{\omega_{\text {lin }}-\omega}{\omega a_{0}^{2} / 2}\right)^{1 / 2}}>0
\end{gathered}
$$

\section{NUMERICAL DATA}

\section{A. Broadening and deformation of the spectrum}

With increasing nonlinearity, the spectrum on axis changes its form starting with more or less visible distortions of the initial linear Gaussian distribution and ending with the appearance of a whole series of secondary maxima at the lower frequency side with modulated amplitude, according to what is shown in Refs. [24,28,33]. Figure 1

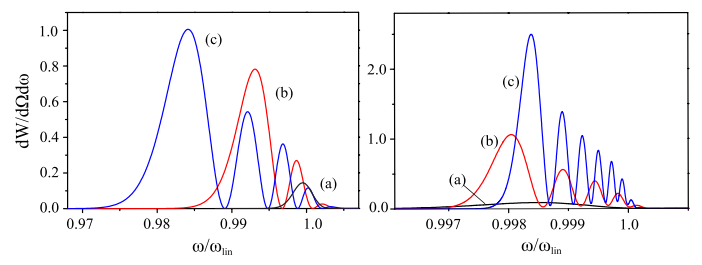

FIG. 1. (left) $\frac{d^{2} W}{d \omega d \Omega}$ as a function of $\omega / \omega_{\text {lin }}$ with increasing laser parameter: (a) $a_{0}=0.015(W=0.05 \mathrm{~J})$, (b) $a_{0}=0.0466(\mathrm{~W}=$ $0.5 \mathrm{~J})$, (c) $a_{0}=0.066(W=1 \mathrm{~J})$. (right) $\frac{d^{2} W}{d \omega d \Omega}$ as a function of $\omega / \omega_{\text {lin }}$ with increasing laser duration $\Delta \tau$ and constant value of $a_{0}=0.0722$ : (a) $\Delta \tau=0.25 \mathrm{ps}\left(W=0.2 \mathrm{~J}, \epsilon=1.5 \times 10^{-3}\right)$, (b) $\Delta \tau=1.5 \mathrm{ps}\left(W=1.2 \mathrm{~J}, \epsilon=2.5 \times 10^{-4}\right)$, (c) $\Delta \tau=3 \mathrm{ps}$ $\left(W=2.4 \mathrm{~J}, \epsilon=1.25 \times 10^{-4}\right)$. Other parameters: $\gamma(0)=200$, $w_{0}=27 \mu \mathrm{m}, \omega_{L} / 2 \pi \approx 6 \times 10^{14} \mathrm{~s}^{-1}$. 
TABLE I. Comparison between the intensity $I_{R}$ deduced by Fig. 5a (blue curve) of Ref. [24] and that calculated by our code $I$ for some points, showing a good agreement.

\begin{tabular}{lrc}
\hline \hline$\omega / \omega_{\text {lin }}$ & \multicolumn{1}{c}{$I_{R}$} & $I$ \\
\hline 1 & $\sim 1900$ & 1823 \\
0.99982 & $\sim 4500$ & 4421 \\
0.9996 & $\sim 0$ & 0.027 \\
0.9992 & $\sim 10200$ & 10083 \\
0.9988 & $\sim 0$ & 0.12 \\
0.99807 & $\sim 17100$ & 16998 \\
0.997 & $\sim 1500$ & 1425 \\
\hline \hline
\end{tabular}

shows examples of the broadening and continuous downshifting of the spectrum in the case of a Gaussian laser pulse. In Table I, we report a comparison between the data that can be deduced by Fig. 5a of Ref. [24], blue curve, and a similar calculation made with our code, showing that the results are in good agreement. The left part of the figure shows the case regarding the increasing of the laser parameter from $a_{0}=0.015(W=0.05 \mathrm{~J})$ to $a_{0}=0.066$ $(W=1 \mathrm{~J})$. While curve (a) presents a quasi-Gaussian shape, several fringes develop as the energy increases [(b) and (c)], together with an evident redshift and spectral enlargement of the radiation.

Increasing the time duration of the laser at fixed $a_{0}$, instead, gives rise to interference fringes whose number depends linearly on $\Delta \tau$, but the overall width and average position of the spectral line do not change, as is shown in Fig. 1 (right).

Figure 2 gives the increase of the relative bandwidth as a function of $a_{0}$ for the same parameters as in Fig. 1. The broadening due to nonlinear effects reaches the level $10^{-3}$ for values of $a_{0}$ larger than 0.5 .

From the expression (35) of the phase $\psi$, one can see that the oscillations in the spectrum come from the modulation of the laser field and increase linearly in number with the laser time duration. The width and the shift of the spectrum, instead, are governed by the function $M$, which depends on $a_{0}$ but is independent of $\Delta \tau$.

Figure 3 presents the dependence of the differential spectrum on both frequency and polar angle $\theta$ and shows

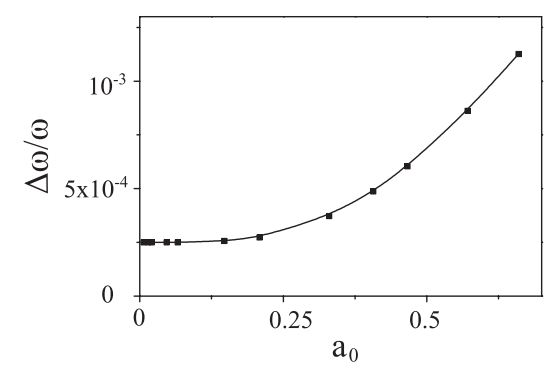

FIG. 2. Rms spectral bandwidth $\Delta \omega / \omega$ on axis as a function of the laser parameter $a_{0}$. Other parameters have the same values as in Fig. 1.

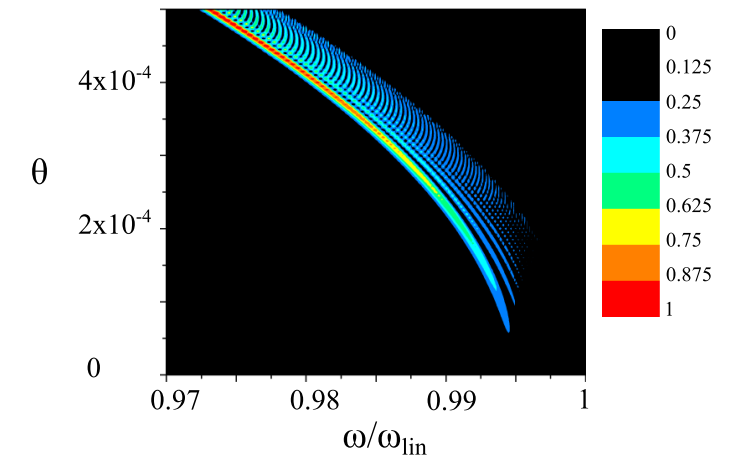

FIG. 3. $\frac{d^{2} W}{d \omega d \Omega} \sin \theta$ as a function of $\omega / \omega_{\text {lin }}$ (abscissa) and $\theta(\mathrm{rad})$ (ordinate) for $\gamma=300$ and $a_{0}=0.125, w_{0}=27 \mu \mathrm{m}$.

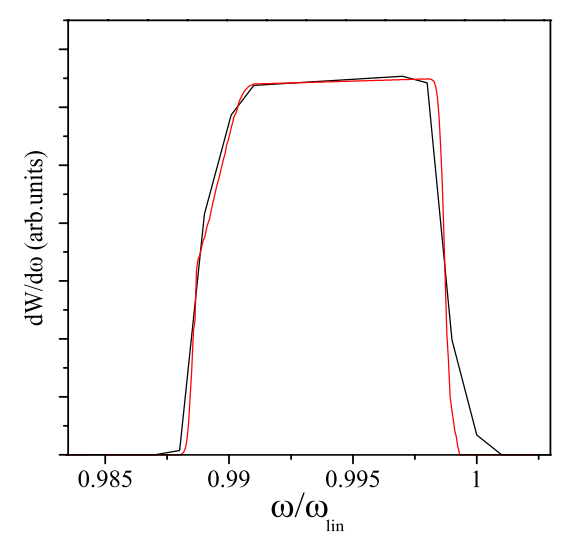

FIG. 4. Angle-integrated spectrum $d W / d \omega$ vs $\omega / \omega_{\text {lin }}$ for $\gamma=200, \theta_{\mathrm{acc}}=500 \mu \mathrm{rad}, a_{0}=0.06, w_{0}=27 \mu \mathrm{m}$. Black curve: nonlinear calculation; red curve: linear calculation.

the correlation between these variables in the nonlinear regime.

The details of the fringes, however, are almost completely absorbed by the broadening of the spectrum connected to the frequency-angular correlation typical of the Thomson/Compton scattering, when integrated over the polar angle $\theta$ from 0 to a final value $\theta_{\text {acc }}$. Figure 4 shows the nonlinear and the linear spectra integrated on a solid angle. The small differences occurring at the edges of the spectrum are further diminished by the integration over the electron beam, with realistic energy spread and emittance, as shown in Ref. [29].

\section{B. Shift of the spectrum}

From Eq. (31) one can see that in the linear case the spectrum is centered around the frequency $\omega_{\text {lin }}$ given in Eq. (16). When nonlinear effects are present, the peak of the spectrum moves backward toward frequencies lower than $\omega_{\text {lin }}$ (redshift), as shown in Fig. 5.

Quantum effects arising from the recoil of the electron in the scattering with the laser photon $[3,4,26,29]$ lead to a spectral redshift that can be neglected for relatively low electron energies or in applications where the required 


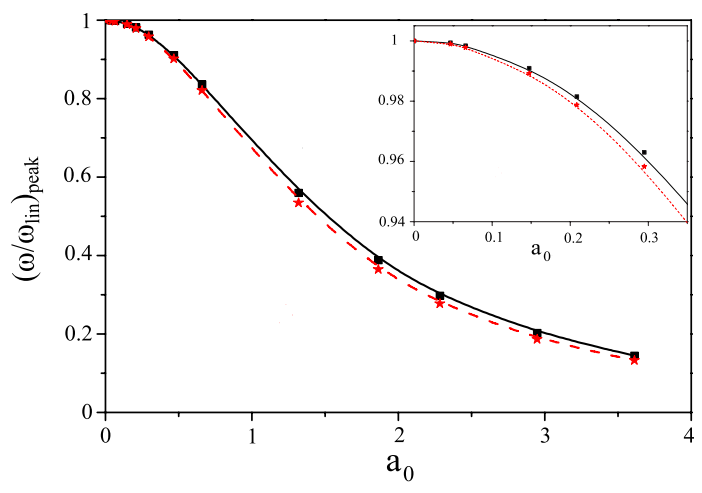

FIG. 5. Position of the peak as a function of $a_{0}$, for the same parameters as Fig. 1. The red curve is the curve $2 /\left(2+a_{0}^{2}\right)$.

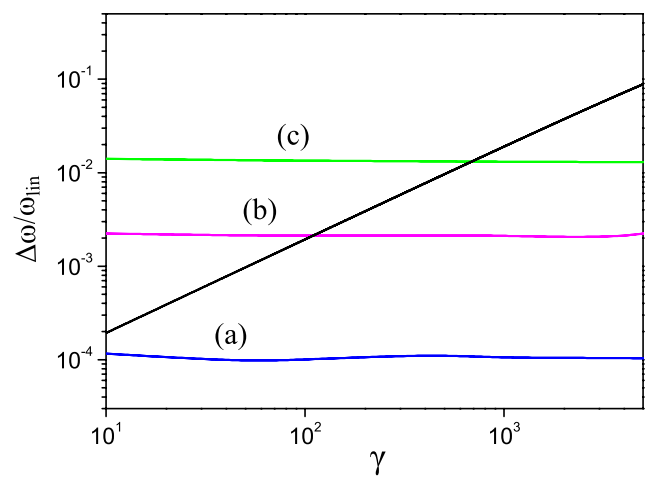

FIG. 6. Comparison between the nonlinear classical redshift and the quantum linear one (black curve): (a) $a_{0}=0.0081$, (b) $a_{0}=0.081$, and (c) $a_{0}=0.18$.

bandwidth is large. However, when the shift is larger than the bandwidth, as in the case of ELI-NP, they have to be taken into account even if they do not seem to affect substantially the shape of the spectrum and the number of photons emitted [24,29]. Figure 6 shows the comparison between the nonlinear shift at different values of $a_{0}$, based on the position of the main peak of the spectrum, and the quantum shift derived through the Compton formula and evaluated in the impact between an electron and the photon of a laser at $500 \mathrm{~nm}$.

As can be seen, for small values of $\gamma$ (less than 100), classical nonlinear effects are comparable and even dominant with respect to the quantum shift. In the intermediate regime, for values of $\gamma$ between 100 and 1000 (i.e., the case of ELI [18]), nonlinear effects are important only when $a_{0}$ has values larger than 0.1 , corresponding to highly compressed laser pulses (which are in general not suitable for Compton experiments) or large laser energy, well beyond the state of the art. For $\gamma \gtrsim 1000$ the quantum shift is always dominant.

\section{A possible experiment on PlasmonX SPARC-LAB}

The occurrence of the nonlinear spectral broadening can be tested on the facility PlasmonX at SPARC-LAB at

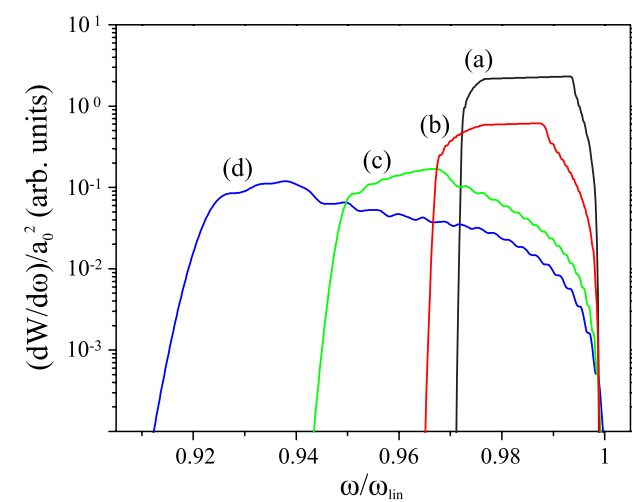

FIG. 7. Angle-integrated spectrum in the case of the experiment PlasmonX at SPARC-LAB for different values of $a_{0}$, obtained by compressing the laser pulse. $\gamma=300, \theta_{\text {acc }}=$ $0.5 \mathrm{mrad}, E_{L}=5 J$, (a) $a_{0}=0.1(\Delta \tau=6 \mathrm{ps})$, (b) $a_{0}=0.15$ $(\Delta \tau=3 \mathrm{ps})$, (c) $a_{0}=0.25(\Delta \tau=1 \mathrm{ps})$, and (d) $a_{0}=0.35$ $(\Delta \tau=0.5 \mathrm{ps})$.

$\gamma=300$. Operating with the laser pulse at total energy of $5 \mathrm{~J}$ and duration $\Delta \tau=6 \mathrm{ps}$, the laser parameter has the value $a_{0}=0.1$ and the nonlinearities do not substantially alter the spectrum, when collected inside an acceptance angle of $1 \mathrm{mrad}$. A compression of the laser pulse up to 1 order of magnitude and in this same condition produces a broadening of the spectrum with a consistent enhancement of the bandwidth. These experimental conditions can be reproduced at SPARC due to the possibility of compressing the laser FLAME [16] reaching in this way powers of $50 \mathrm{TW}$, operating at $5 \mathrm{~J}$ in the range from $10 \mathrm{ps}$ up to $100 \mathrm{fs}$, with values of the laser parameters $a_{0}$ from 0.1 to 0.36, as shown in Fig. 7. The use of a value of the electron Lorentz factor of about $\gamma=300$ will permit also the control of the validity of the classical model.

\section{CONCLUSIONS}

The nonlinear effects have been analyzed by means of a numerical analysis and of analytical expressions of the double differential spectrum based on the stationary phase method. While the on-axis spectrum for a single electron may in some cases be very different from the linear Gaussian shape, when the contributions are summed on the solid angle, modest variations have been found for energetic electron beams in the cases considered, with shifts of the wavelength amply inside the bandwidths and small enlargement of the spectrum.

[1] M. Bech, O. Bunk, C. David, R. Ruth, J. Rifkin, R. Loewen, R. Feidenhans'1, and F. Pfeiffer, J. Synchrotron Radiat. 16, 43 (2009).

[2] M. Babzien et al., Phys. Rev. Lett. 96, 054802 (2006).

[3] D. J. Gibson, F. Albert, S. G. Anderson, S. M. Betts, M. J. Messerly, H. Phan, V.A. Semenov, M. Shverdin, A. M. 
Tremaine, F. Hartemann et al., Phys. Rev. ST Accel. Beams 13, 070703 (2010).

[4] C. Sun and Y. K. Wu, Phys. Rev. ST Accel. Beams 14, 044701 (2011).

[5] J. Miao et al., Proc. Natl. Acad. Sci. U.S.A. 100, 110 (2003).

[6] H. N. Chapman et al., Nat. Phys. 2, 839 (2006).

[7] T. Weitkamp, A. Diaz, C. David, F. Pfeiffer, M. Stampanoni, P. Cloetens, and E. Ziegler, Opt. Express 13, 6296 (2005).

[8] T. Shintake et al., Nat. Photonics 2, 555 (2008).

[9] Z. Huang et al., Phys. Rev. ST Accel. Beams 13, 020703 (2010).

[10] W. Ackermann et al., Nat. Photonics 1, 336 (2007).

[11] L. Giannessi et al., Phys. Rev. Lett. 108, 164801 (2012).

[12] J. Mauritsson, P. Johnsson, R. López-Martens, K. Varjú, W. Kornelis, J. Biegert, U. Keller, M. Gaarde, K. Schafer, and A. L'Huillier, Phys. Rev. A 70, 021801(R) (2004).

[13] P. Tomassini, A. Giulietti, D. Giulietti, and L. Gizzi, Appl. Phys. B 80, 419 (2005).

[14] P. Tomassini et al., IEEE Trans. Plasma Sci. 36, 1782 (2008).

[15] P. Oliva, A. Bacci, U. Bottigli, M. Carpinelli, P. Delogu, M. Ferrario, D. Giulietti, B. Golosio, V. Petrillo, L. Serafini et al., Nucl. Instrum. Methods Phys. Res., Sect. A 615, 93 (2010).

[16] L. Gizzi, A. Bacci, S. Betti et al., Eur. Phys. J. Special Topics 175, 3 (2009).

[17] L. Giannessi, Phys. Rev. ST Accel. Beams 14, 060712 (2011).
[18] European proposal for the Compton gamma-ray of ELINP, http://www.e-gammas.com.

[19] A. Bacci, M. Ferrario, C. Maroli, V. Petrillo, and L. Serafini, Phys. Rev. ST Accel. Beams 9, 060704 (2006).

[20] J. Jackson, Classical Electrodynamics (John Wiley and Sons, New York, 1975), 2nd ed.

[21] E. Esarey, S. K. Ride, and P. Sprangle, Phys. Rev. E 48, 3003 (1993).

[22] S. K. Ride, E. Esarey, and M. Baine, Phys. Rev. E 52, 5425 (1995).

[23] Y. Lau, F. He, D. P. Umstadter, and R. Kowalczyk, Phys. Plasmas 10, 2155 (2003).

[24] F. Albert et al., Phys. Rev. ST Accel. Beams 13, 070704 (2010).

[25] D. Seipt and B. Kampfer, Phys. Rev. ST Accel. Beams 14, 040704 (2011).

[26] F. Hartemann, Nucl. Instrum. Methods Phys. Res., Sect. A 608, S1 (2009).

[27] F. He, Y. Y. Lau, D. P. Umstadter, and R. Kowalczyk, Phys. Rev. Lett. 90, 055002 (2003).

[28] M. Boca and V. Florescu, Eur. Phys. J. D 61, 449 (2011).

[29] V. Petrillo et al., Nucl. Instrum. Methods Phys. Res., Sect. A 693, 109 (2012).

[30] M. V. Klein and T. E. Furtak, Optics (John Wiley and Sons, New York, 1986), 2nd ed.

[31] A. Erdely, Asymptotic Expansions, California Institute of Technology (Dover Publications, New York, 1956).

[32] E. T. Copson, Asymptotic Expansions (Cambridge University Press, London, 1965).

[33] F. Hartemann, F. Albert, C. Siders, and C. Barty, Phys. Rev. Lett. 105, 130801 (2010). 